\title{
Análise da ocorrência de incidentes notificados no ambiente hospitalar de uma maternidade pública
}

\author{
Analysis of reported incidents in the hospital setting of a public maternity hospital
}

Análisis los incidentes reportados en un hospital público de maternidad

Wenderson Costa da Silva ${ }^{1 *}$, Hálmisson D’Árley Santos Siqueira ${ }^{1}$, Alanna Nunes Soares ${ }^{1}$, Rafael Andrade da Silva ${ }^{1}$, Chrisllayne Oliveira da Silva ${ }^{1}$, Karine Costa Melo ${ }^{1}$, Layse Siqueira Costa Miranda ${ }^{1}$, Laila da Silva Mota ${ }^{1}$, Rogério Cruz Mendes ${ }^{1}$, César Augusto Pereira Souza Filho ${ }^{1}$, Elide Andressa de Andrade Rodrigues Severo ${ }^{1}$, Eduardo Brito da Silva ${ }^{1}$, Samuel de Jesus de Melo Silva ${ }^{1}$, Thalia Jeovana da Silva Pereira ${ }^{1}$, Alanna Sanlai Sousa Lima ${ }^{1}$.

\section{RESUMO}

Objetivo: Determinar a prevalência de incidentes notificados no ambiente hospitalar de uma maternidade pública. Métodos: Tratou-se de uma pesquisa de análise documental, retrospectiva, do tipo descritivaexploratória, com uma abordagem quantitativa, realizada com 166 fichas de incidentes notificados de uma maternidade pública, localizada no município de Caxias-MA. Resultados: Foi possível constatar que a maioria dos incidentes foram erros relacionados à identificação do paciente $(22,3 \%)$, e nenhum incidente de segurança foi associado à mortalidade. As mães configuraram como o grupo mais prevalente de casos de incidentes $(66,9 \%)$, e a fase com maior acontecimento desses eventos foi durante o período de internação $(75,3 \%)$. A maior prevalência de casos notificados se concentrou no período de março de 2018 a fevereiro de 2019 (68,1\%). Conclusão: Os resultados reforçam a necessidade de estratégias, como de educação permanente da equipe de saúde, capacitação a partir de protocolos e guias de procedimentos para minimizar seus índices e estimular o monitoramento contínuo e preenchimento correto das fichas. A gestão hospitalar deve demonstrar apoio aos seus profissionais, desmistificando que esses incidentes não são um subproduto inevitável da assistência à saúde, mas passiveis de prevenção, através de uma cultura colaborativa, sem punição e a valorização dos profissionais que as notificam.

Palavras-chave: Segurança do Paciente, Notificação, Incidentes, Hospital maternidade.

\section{ABSTRACT}

Objective: To determine the prevalence of reported incidents in the hospital setting of a public maternity hospital. Methods: This was a descriptive-exploratory, retrospective documentary analysis research with a quantitative approach, carried out with 166 reports of reported incidents from a public maternity hospital, located in Caxias-MA. Results: It was found that most of the incidents were errors related to patient identification $(22.3 \%)$, and no safety incident was associated with mortality. Mothers were the most prevalent group of incident cases $(66.9 \%)$, and the phase with the highest occurrence of these events was during the hospitalization period (75.3\%). The highest prevalence of reported cases was from March 2018 to February 2019 (68.1\%). Conclusion: The results reinforce the need for strategies, such as permanent education of the Equipe de Saúde, training based on protocols and procedural guidelines to minimize their indexes and stimulate or continuous monitoring and correct preenchimento das chips. A hospital gesture must show support for your six professionals, demystifying that these incidents are not a subproduto inevitável da assistência à saúde, more passiveis de prevenção, through a collaborative culture, sem punição e a valorização dos profissionais que so notifies.

Keywords: Patient Safety, Notification, Incidents, Maternity hospital.

${ }^{1}$ Centro Universitário de Ciências e Tecnologia do Maranhão (UniFacema). Caxias - MA.

*E-mail: wendersoncosta09@hotmail.com

SUBMETIDO EM: 8/2019 


\section{RESUMEN}

Objetivo: determinar la prevalencia de incidentes reportados en el entorno hospitalario de una maternidad pública. Métodos: Se realizó una investigación descriptiva-exploratoria de análisis documental retrospectivo con un enfoque cuantitativo, realizada con 166 informes de incidentes notificados desde un hospital público de maternidad, ubicado en Caxias-MA. Resultados: se encontró que la mayoría de los incidentes fueron errores relacionados con la identificación del paciente (22.3\%), y ningún incidente de seguridad se asoció con mortalidad. Las madres fueron el grupo más frecuente de casos incidentes $(66,9 \%)$, y la fase con la mayor ocurrencia de estos eventos fue durante el período de hospitalización $(75,3 \%)$. La prevalencia más alta de casos reportados fue de marzo de 2018 a febrero de 2019 (68,1\%). Conclusión: Los resultados de la reforma son una necesidad de estrategias, como la educación permanente del equipo de seguridad, la capacidad a partir de protocolos y guías de procedimientos para minimizar los índices y el control continuo y el preajuste correcto de las fichas. A gestão hospitalar deve demonstrar apoio aos seus profissionais, desmistificando que esses incidentes não são um subproduto inevitável da assistência à saúde, mas passiveis de prevenção, através de uma cultura colaborativa, sem punição e a valorização dos profissionais que as notificam.

Palabras clave: Seguridad del paciente, Notificación, Incidencias, Hospital de maternidad.

\section{INTRODUÇÃO}

De acordo com a Classificação Internacional de Segurança do Paciente (CISP) da Organização Mundial da Saúde (OMS), um incidente é um evento ou situação que poderia ter causado, ou causou, um dano desnecessário ao cliente. Classificam-se em: Circunstância Notificável - Incidente com potencial dano ou lesão; Near miss (quase erro) - Incidente que não chegou a afetar o paciente; Incidente sem lesão - Incidente que atingiu o paciente, mas não resultou danos; e Evento Adverso (EA) - Incidente que causou danos ao paciente (WHO, 2009).

Estudos contabilizam que a ocorrência de incidentes associados à assistência à saúde atinja de $4,0 \%$ a $16 \%$ de pacientes hospitalizados em países desenvolvidos, o que impactou sistemas de saúde de diversos países a melhorar a segurança do cliente frente aos serviços de saúde (BRASIL, 2017). Estima-se que em todo o mundo aconteçam todo ano 43 milhões de EA, provocando 23 milhões de anos de vida perdidos por incapacidade (ADHIKARI NKJ, 2013). No Brasil, estudo realizado em três hospitais do Rio de Janeiro a incidência de pacientes com EA foi de 7,6\% (84 de 1103 pacientes) (MENDES W, et al., 2009).

Os fatores que contribuem são circunstanciais, ações ou influências que operam papel na origem ou no desenvolvimento de um incidente, como: não seguir um protocolo da instituição, imperícia do profissional que cuida, ausência de comunicação entre os profissionais e dessa com o cliente, entre outros (SOUSA P e MENDES W, 2014). De acordo com Fassini P e Hahn GV (2012), cabe aos profissionais detectar os riscos, garantir a segurança dos pacientes, bem como, evitar ou diminuir as intercorrências durante sua estadia na instituição.

Com base no exposto, traçou-se a seguinte problemática: quais os incidentes mais recorrentes notificados no ambiente hospitalar de uma maternidade pública localizada no município de Caxias-MA? O objetivo geral dessa pesquisa foi determinar a prevalência de incidentes notificados no ambiente hospitalar de uma maternidade pública.

Especificamente, objetivou-se: identificar os incidentes mais comuns notificados no ambiente hospitalar da maternidade; determinar o perfil dos pacientes que sofrem eventos adversos; e identificar a fase da assistência em que ocorrem os maiores números de casos de incidentes notificados.

\section{MÉTODOS}

O presente estudo tratou-se de uma pesquisa de análise documental, retrospectiva, do tipo descritivaexploratória, com uma abordagem quantitativa. A pesquisa foi realizada em uma maternidade pública, localizada no município de Caxias-MA. A Maternidade é referência para 06 municípios da região de saúde de Caxias e para mais de outros 50 municípios da Macrorregião. Possui 75 leitos, sendo 10 de UTI, e realiza o trabalho de média e alta complexidade. A escolha dessa instituição deveu-se ao fato de ser a única instituição 
municipal atual hospitalar pública há aderir ao Programa de Segurança do Paciente (PSP) com um Núcleo de Segurança do Paciente (NSP) implantado.

O universo do estudo foi composto pelas fichas de notificações de incidentes do núcleo de segurança do paciente da maternidade em que a pesquisa foi realizada. A coleta de dados na instituição resultou de um total de 166 fichas de notificações de incidentes notificados.

Foram incluídas no estudo todas as fichas de incidentes registrados devidamente preenchidas e armazenadas, em perfeitas condições, datadas entre o período de março de 2017 a março de 2019, totalizando 2 anos.

Foram excluídos da amostra os documentos com problemas no conteúdo, escrita ilegível, rasuras ou por estarem danificados, e a credibilidade das informações neles contidos (se não continham omissões, erros ou distorções), além daquelas fora do recorte temporal. O critério que levou à determinação do recorte temporal foi o fato de que o núcleo de segurança do paciente foi criado em março de 2017 , momento quando os casos de incidentes e eventos adversos passaram a ser notificados.

Os dados da pesquisa foram coletados das fichas de notificações do núcleo de segurança da maternidade, no período de abril e maio de 2019. A informações dos documentos foram extraídas através de um instrumento de coleta de dados, contendo questões relativas ao tema do estudo, como: dados sociodemográficos; data de internação, de notificação e do evento adverso; identificação dos incidentes; etc.

A análise dos resultados obtidos foi processada por intermédio de cálculos estatísticos, colocados em números absolutos e percentuais, da forma que melhor demonstrasse a quantidade amostral. Os dados foram armazenados em planilha do programa Microsoft Office Exce/[ for Windows $\mathbb{B}^{\circledR}$ e dispostos em tabelas e gráfico de análise descritiva.

O Projeto de pesquisa foi apreciado e devidamente aprovado pela Secretaria Municipal de Saúde de Caxias - MA. Em seguida, foi submetido à Plataforma Brasil e direcionado ao Comitê de Ética em Pesquisa do Centro Universitário de Ciências e Tecnologia do Maranhão (CEP/UniFACEMA). E aprovado com № de CAAE 09545019.5.0000.8007.

O desenvolvimento do estudo seguiu todos os procedimentos éticos de pesquisa, pleiteando as técnicas adequadas descritas na literatura para o cumprimento dos preceitos éticos relacionados à pesquisa com documentos, estabelecidos pela resolução 466/12 do Conselho Nacional de Saúde (CNS). Assim sendo, a coleta de dados desse estudo somente foi realizada após ter sido aprovada pelo Comitê de Ética em Pesquisa (CEP).

\section{RESULTADOS}

A coleta de dados na instituição resultou de um total de 166 fichas de notificações de incidentes. Quanto ao perfil dos pacientes identificados o grupo com maior número de casos notificados foram as mães, correspondente a $111(66,9 \%)$, sendo $32(28,8 \%)$ delas com faixa etária entre 21 a 30 anos. Já os recémnascidos foram 38 (22,9\%) notificações, sendo que 17 (44,7\%) deles eram do sexo feminino e 25 (65,8\%) com faixa etária entre um a sete dias de nascido.

Dentre as variáveis analisadas na Tabela 1, os erros de identificação do paciente foram os eventos mais relatados pelos profissionais nas fichas de notificação de incidentes, correspondendo a $37(22.3 \%)$ do total levantado. Em seguida, outros eventos foram os mais notificados pelos profissionais; estes outros referiamse a: omissão $(16,9 \%)$, quedas em pacientes $(10,8 \%)$, falta de material médico hospitalar $(8,4 \%)$ e flebite (6,6\%). Em relação à omissão ao paciente, $60,7 \%$ ocorreram por omissão de dose e $39,3 \%$ por omissão de cuidado. A fase mais prevalente dos acontecimentos dos eventos notificados foi durante o período de internação $(75,3 \%)$. Em relação aos setores responsáveis pela análise das ocorrências, apresentaram maior número de notificações a clínica obstétrica $(45,8 \%)$, seguida do pré-parto $(19,3 \%)$ e a Unidade de Terapia Intensiva Neonatal (UTIN) (15,1\%). Em 62\% dos casos, o turno de maior ocorrência foi ignorado nos registros das fichas. Assim como em $53,0 \%$ dos registros a categoria responsável pela notificação foi ignorada. 
Tabela 1 - Número e percentual de incidentes, segundo as características, fase da assistência, setor, turno e categoria profissional. Caxias-MA, $2019(\mathrm{n}=166)$.

\begin{tabular}{|c|c|c|}
\hline Variáveis & $\mathbf{N}$ & $\%$ \\
\hline \multicolumn{3}{|l|}{ Incidentes } \\
\hline Erros relacionados a identificação do paciente & 37 & 22,3 \\
\hline Omissão & 28 & 16,9 \\
\hline Quedas em paciente & 18 & 10,8 \\
\hline Falta de material médico hospitalar & 14 & 8,4 \\
\hline Flebite & 11 & 6,6 \\
\hline Evasão de pacientes & 9 & 5,4 \\
\hline Quase Evento & 8 & 4,9 \\
\hline Reações adversas a medicação & 6 & 3,6 \\
\hline Farmacovigilância (medicamentos e saneantes) & 5 & 3,0 \\
\hline Falha a assistência à saúde & 5 & 3,0 \\
\hline Lesão por pressão & 5 & 3,0 \\
\hline Risco relacionado ao ambiente & 4 & 2,4 \\
\hline Extubação Acidental & 4 & 2,4 \\
\hline Sofrimento (físico, social ou psicológico) & 3 & 1,8 \\
\hline Remoção não programada de cateter, sonda, dreno, tubo, etc. & 3 & 1,8 \\
\hline Medicação com desvio de qualidade & 2 & 1,2 \\
\hline Tecnovigilância (material médico hospitalar/equipamento defeituoso & 2 & 1,2 \\
\hline Erro relacionado ao procedimento cirúrgico & 1 & 0,6 \\
\hline Erro relacionado à transfusão de hemoderivado & 1 & 0,6 \\
\hline Total & 166 & 100,0 \\
\hline \multicolumn{3}{|l|}{ Omissão } \\
\hline Omissão de dose & 17 & 60,7 \\
\hline Omissão de cuidado & 11 & 39,3 \\
\hline Total & 28 & 100,0 \\
\hline \multicolumn{3}{|l|}{ Fase da Assistência } \\
\hline Admissão & 21 & 12,7 \\
\hline Internação & 125 & 75,3 \\
\hline Alta & 3 & 1,8 \\
\hline Ignorado & 17 & 10,2 \\
\hline Total & 166 & 100,0 \\
\hline \multicolumn{3}{|l|}{ Setor } \\
\hline Clínica Obstétrica & 76 & 45,8 \\
\hline Pré-parto & 32 & 19,3 \\
\hline Unidade de Terapia Intensiva Neonatal & 25 & 15,1 \\
\hline Unidade de Cuidado Intermediário Neonatal Convencional & 6 & 3,6 \\
\hline Classificação de risco & 3 & 1,8 \\
\hline Sala de Recuperação Pós-Anestésica & 3 & 1,8 \\
\hline Unidade de Cuidado Intermediário Neonatal Canguru & 3 & 1,8 \\
\hline Alojamento conjunto & 2 & 1,2 \\
\hline Centro Cirúrgico & 2 & 1,2 \\
\hline Ignorado & 15 & 9,0 \\
\hline Total & 166 & 100,0 \\
\hline \multicolumn{3}{|l|}{ Turno } \\
\hline Manhã & 30 & 18,1 \\
\hline Tarde & 22 & 13,3 \\
\hline Noite & 11 & 6,6 \\
\hline Ignorado & 103 & 62,0 \\
\hline Total & 166 & 100,0 \\
\hline \multicolumn{3}{|l|}{ Categoria profissional } \\
\hline Enfermagem & 77 & 46,4 \\
\hline Médico & - & - \\
\hline Nutricionista & - & - \\
\hline Fisioterapeuta & - & - \\
\hline Psicólogo & - & - \\
\hline Assistente social & - & - \\
\hline Técnico em enfermagem & 1 & 0,6 \\
\hline Auxiliar em enfermagem & - & - \\
\hline Ignorado & 88 & 53,0 \\
\hline Total & 166 & 100,0 \\
\hline
\end{tabular}

Legenda: N=número; \%=percentual. Fonte: Silva WC, et al. 2019. 
Quanto aos Incidentes maternos e os setores de ocorrência, os erros relacionados a identificação da paciente $(27 \%)$ apresentaram um maior percentual, seguido por omissão $(20,7 \%)$ e quedas $(15,3 \%)$. Entre os setores destacam-se a clínica obstétrica $(62,1 \%)$ e o pré-parto $(28,8 \%)$, conforme demonstrado na Tabela 2.

Tabela 2 - Número e percentual dos Incidentes segundo a ocorrência com as mães. Caxias - MA, 2019. $(\mathrm{n}=111)$.

\begin{tabular}{|c|c|c|}
\hline Variáveis & $\mathbf{N}$ & $\%$ \\
\hline \multicolumn{3}{|l|}{ Incidentes } \\
\hline Erros relacionados a identificação da paciente & 30 & 27,0 \\
\hline Omissão & 23 & 20,7 \\
\hline Quedas em pacientes & 17 & 15,3 \\
\hline Evasão de pacientes & 8 & 7,2 \\
\hline Quase evento & 6 & 5,4 \\
\hline Falha a assistência à saúde & 5 & 4,5 \\
\hline Flebite & 4 & 3,6 \\
\hline Reações adversas a medicação & 4 & 3,6 \\
\hline Sofrimento (físico, social ou psicológico) & 3 & 2,7 \\
\hline Falta de material médico hospitalar & 3 & 2,7 \\
\hline Remoção não programada de cateter, sonda, dreno, tubo, etc. & 2 & 1,8 \\
\hline Tecnovigilância (material médico hospitalar/equipamento defeituoso & 1 & 0,9 \\
\hline Riscos relacionados ao ambiente & 1 & 0,9 \\
\hline Famarcovigilância (medicamentos e seneantes) & 1 & 0,9 \\
\hline Lesão por pressão & 1 & 0,9 \\
\hline Erro relacionado ao procedimento cirúrgico & 1 & 0,9 \\
\hline Erro relacionado à transfusão de hemoderivado & 1 & 0,9 \\
\hline Total & 111 & 100,0 \\
\hline \multicolumn{3}{|l|}{ Setor } \\
\hline Clínica Obstétrica & 69 & 62,1 \\
\hline Pré-parto & 32 & 28,8 \\
\hline Classificação de risco & 3 & 2,7 \\
\hline Sala de Recuperação Pós-Anestésica & 3 & 2,7 \\
\hline Alojamento conjunto & 2 & 1,8 \\
\hline Centro Cirúrgico & 2 & 1,8 \\
\hline Total & 111 & 100,0 \\
\hline
\end{tabular}

Legenda: N=número; \%=percentual. Fonte: Silva WC, et al. 2019.

Entre os incidentes analisados com os Recém-Nascido (RN) e os setores de ocorrência, a Tabela 3 explana os que mais ocorreram, sendo eles os erros relacionados a identificação dos $\mathrm{RN}$ e flebite com $18,4 \%$ cada, e omissão (13,2\%).

As notificações dos eventos lesão por pressão, extubação acidental e a falta de material médico hospitalar obtiveram o mesmo percentual de ocorrência, com 10,5\% cada. E o setor com maior número de casos notificados foi a UTIN $(65,8 \%)$. 
Tabela 3 - Número e percentual dos Incidentes segundo a ocorrência com os recém-nascidos. Caxias - MA, 2019. $(n=38)$.

\begin{tabular}{lcc}
\hline Variáveis & N & $\%$ \\
\hline Incidentes no recém-nascido & & \\
Erros relacionados a identificação do paciente & 7 & 18,4 \\
Flebite & 7 & 18,4 \\
Omissão & 5 & 13,2 \\
Lesão por pressão & 4 & 10,5 \\
Extubação Acidental & 4 & 10,5 \\
Falta de material médico hospitalar & 4 & 10,5 \\
Quase evento & 2 & 5,3 \\
Reações adversas à medicação & 2 & 5,3 \\
Quedas em paciente & 1 & 2,6 \\
Evasão de pacientes & 1 & 2,6 \\
Remoção não programada de cateter, sonda, dreno, tubo, etc. & 1 & 2,6 \\
Total & 38 & 100,0 \\
Setor & & \\
Clínica Obstétrica & 4 & 10,5 \\
Unidade de Cuidado Intermediário Neonatal Canguru & 3 & 7,9 \\
Unidade de Cuidado Intermediário Neonatal Convencional & 6 & 15,8 \\
Unidade de Terapia Intensiva Neonatal & 25 & 65,8 \\
Total & 38 & 100,0 \\
\hline
\end{tabular}

Legenda: N=número; \%=percentual.

Fonte: Silva WC, et al. 2019.

A Tabela 4 apresenta os meses e anos em que ocorreram incidentes notificados no ambiente hospitalar da maternidade. O ano com maior prevalência de casos notificados foi o de 2018 (63,3\%); o mês mais prevalente foi o de maio $(19,0 \%)$ e o com menor ocorrência foi o de setembro (3,8\%). Todavia, no ano de 2017, o núcleo de segurança do paciente foi implantado somente em março, quando oficialmente as notificações já poderiam ser registradas; e o ano de 2019 ainda não foi concluído; dessa forma, estima-se um aumento em sua prevalência.

Tabela 4 - Distribuição dos meses e anos em que ocorreram incidentes e eventos adversos notificados na maternidade. Caxias-MA, $2019(n=166)$.

\begin{tabular}{lcccccccc}
\hline \multirow{2}{*}{ Mês de ocorrência } & \multicolumn{8}{c}{ Ano de ocorrência } \\
\cline { 2 - 10 } & $\mathbf{2}$ & $\mathbf{2 0 1 7}$ & $\mathbf{2 0 1 8}$ & $\mathbf{2 0 1 9}$ & \multicolumn{2}{c}{ Total } \\
& - & - & 8 & 7,6 & 8 & 36,4 & 16 & $\%$ \\
\hline Janeiro & - & - & 6 & 5,7 & 14 & 63,6 & 15 & 12,0 \\
Fevereiro & - & - & - & - & - & - & - & - \\
Março & - & - & 8 & 7,6 & - & - & 8 & 4,8 \\
Abril & - & - & 20 & 19,0 & - & - & 20 & 12,0 \\
Maio & 3 & 7,7 & 6 & 5,7 & - & - & 9 & 5,4 \\
Junho & 6 & 15,4 & 14 & 13,3 & - & - & 20 & 12,0 \\
Julho & 8 & 20,5 & 9 & 8,6 & - & - & 17 & 10,2 \\
Agosto & 1 & 2,6 & 4 & 3,8 & - & - & 5 & 3,0 \\
Setembro & 7 & 17,9 & 8 & 7,6 & - & - & 15 & 9,0 \\
Outubro & 10 & 25,6 & 11 & 10,5 & - & - & 21 & 12,7 \\
Novembro & 4 & 10,3 & 11 & 10,5 & - & - & 15 & 9,0 \\
Dezembro & 39 & 23,5 & 105 & 63,3 & 22 & 13,3 & 166 & 100,0 \\
Total & & 79 & & & & & \\
\hline
\end{tabular}

Legenda: $\mathrm{N}=$ número; \%=percentual.

Fonte: Silva WC, et al. 2019.

O Gráfico 1 mostra o crescimento de incidentes notificados que ocorreram nos anos de 2017 e 2018, entre os meses de março a dezembro de ambos os anos. Foi possível constatar um aumento no número de notificações, onde em 2017 foi de 39 (30\%) e em 2018 de 91 (70\%). Houve um aumento de 40\% das notificações só em 2018, comparado ao ano de 2017. 
Gráfico 1 - Distribuição do percentual de incidentes e eventos entre os meses de março a dezembro do ano de 2017 e 2018. Caxias-MA, 2019 ( $n=130)$.

\section{Percentagem de crescimento de incidentes ou eventos adversos dos meses de março a dezembro do ano de 2017 e 2018}

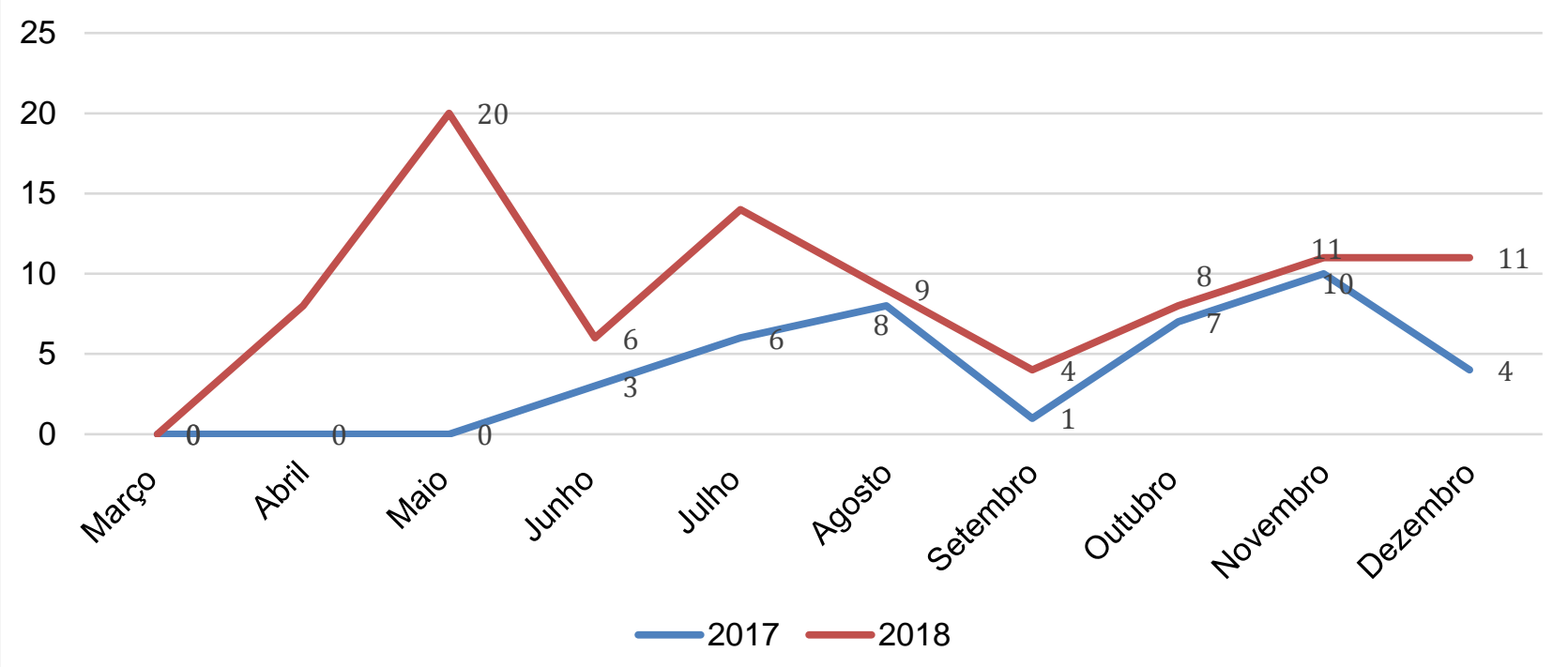

Fonte: Silva WC, et al. 2019.

Em relação à prevalência dos casos notificados que ocorreram nos últimos dois anos, observou-se que o número de notificações aumentou com o decorrer dos anos, sendo de $31,9 \%$ no primeiro ano de março de 2017 a fevereiro de 2018 , para $68,10 \%$ do período de março de 2018 a fevereiro de 2019 . Assim, houve um aumento de $36,2 \%$ de casos notificados no período de um ano, conforme observado na tabela 5.

Tabela 5 - Prevalência de incidentes que aconteceram nos últimos dois anos da amostra estudada. CaxiasMA, $2019(n=166)$.

\begin{tabular}{|c|c|c|c|c|c|c|}
\hline \multirow{3}{*}{ Mês de ocorrência } & \multicolumn{6}{|c|}{ Ano de ocorrência } \\
\hline & \multicolumn{2}{|c|}{ 2017-2018 } & \multicolumn{2}{|c|}{ 2018-2019 } & \multicolumn{2}{|c|}{ Total } \\
\hline & $\mathbf{N}$ & $\%$ & $\mathbf{N}$ & $\%$ & $\mathbf{N}$ & $\%$ \\
\hline Marco & - & - & - & - & - & - \\
\hline Abril & - & - & 8 & 7,1 & 8 & 4,8 \\
\hline Maio & - & - & 20 & 17,7 & 20 & 12,0 \\
\hline Junho & 3 & 5,7 & 6 & 5,3 & 9 & 5,4 \\
\hline Julho & 6 & 11,3 & 14 & 12,4 & 20 & 12,0 \\
\hline Agosto & 8 & 15,1 & 9 & 8,0 & 17 & 10,2 \\
\hline Setembro & 1 & 1,9 & 4 & 3,5 & 5 & 3,0 \\
\hline Outubro & 7 & 13,2 & 8 & 7,1 & 15 & 9,0 \\
\hline Novembro & 10 & 18,9 & 11 & 9,7 & 21 & 12,7 \\
\hline Dezembro & 4 & 7,5 & 11 & 9,7 & 15 & 9,0 \\
\hline Janeiro & 8 & 15,1 & 8 & 7,1 & 16 & 9,6 \\
\hline Fevereiro & 6 & 11,3 & 14 & 12,4 & 20 & 12,0 \\
\hline Total & 53 & 31,9 & 113 & 68,1 & 166 & 100,0 \\
\hline
\end{tabular}

Legenda: $\mathrm{N}=$ número; \%=percentual.

Fonte: Silva WC, et al. 2019.

\section{DISCUSSÃO}

A coleta de dados na instituição resultou num total de 166 fichas de incidentes notificados. Com isso, podese avaliar o número de notificações de acordo com o tipo de evento levantado durante todo o período analisado. Foi possível constatar que a maioria dos incidentes foram os erros relacionados à identificação do 
paciente, e nenhum incidente de segurança foi associado à mortalidade. $O$ grupo mais prevalente foi o das mães, e a fase com maior acontecimento desses eventos foi durante o período de internação. A maior prevalência de casos notificados se concentrou no período de março de 2018 a fevereiro de 2019.

No que tange às fichas analisadas neste estudo, a maioria dos eventos identificados concentrou-se as mães com faixa etária de 20 a 30 anos. Os achados relacionados às características sociodemográficas desta pesquisa corroboram com outros estudos similares. No estudo realizado por Oliveira TC (2018), com prontuários em maternidades do estado de Alagoas, também houve um maior número de casos nesta faixa etária de idade. Esses dados apresentam similaridades nos achados de Morse ML, et al. (2011). A literatura Internacional na área também revela essa prevalência.

O estudo de Adeoye IA, et al. (2013), conduzido nas maternidades de Hospitais Universitários da Nigéria, evidenciou que $41 \%$ dos incidentes ocorreram na faixa etária de 20 a 29 anos. Em Cuba, o estudo conduzido por Mourelle JAF, et al. (2015) e em Moçambique por David E, et al. (2014), apresentaram resultados semelhantes.

No tocante aos incidentes que mais ocorreram com os $\mathrm{RN}$ e mães, os resultados mostram que grande parte das falhas ocorreram durante a identificação do paciente; com as mães foi seguido de omissão e quedas. No entanto, a literatura cientifica brasileira tem reportado que os principais incidentes estão associados à cadeia medicamentosa, como no estudo de Munhoz OL, et al. (2018), realizado em um hospital universitário de grande porte do Rio Grande do Sul, onde consta que 63.6\% estava associado a medicamentos. Resultados semelhantes ao de Souza RF, et al. (2018) $(29,6 \%)$ na cidade de Petrolina, Figueiredo ML, et al. (2018) (18,9\%) em Montes Claros - Minas Gerais e Silva LA, et al. (2014) (63\%) no Sul de Minas Gerais.

A incorreta identificação do paciente é um problema reconhecido mundialmente e uma fonte de erros durante a assistência à saúde, principalmente as falhas de medicação, como observado nos registros das fichas, cujos efeitos são desastrosos ao cliente, profissional e a instituição de saúde. A pesquisa de Tase TH, et al. (2018), realizada em uma maternidade, constatou que as gestantes admitidas na unidade obstétrica partilham riscos para as falhas na identificação, resultantes da semelhança ou grafia idêntica ou na pronunciação de nome ou sobrenome, em conformidade da similaridade do som. Nesse sentido, reitera-se a indispensabilidade do emprego de outros identificadores.

Segundo Souza FT, et al. (2014), o maior problema na identificação do paciente vem de cunho institucional, no qual a ausência de suporte adequado para a identificação correta do paciente na instituição provoca esses erros. Podem ser citados a ausência de pulseiras de identificação, espaço físico reduzido, ausência de placa de identificação nos leitos, etc.

No que se refere à omissão ao paciente, $60,7 \%$ foram relacionados à omissão de dose, por adiamento, erro de prescrição médica ou o medicamento não estava disponível na instituição. Quanto à omissão de cuidados, como a não realização de teste glicemia, ausência da verificação de sinais vitais, assistência negada, Sistematização da Assistência de Enfermagem (SAE) não realizada, entre outros, ocorreram em $39,3 \%$ dos casos.

De acordo com Anacleto TA, et al. (2010), as falhas de comunicação são fatores importantes de falhas de medicação. As instituições de saúde precisam promover a diminuição das barreiras de comunicação entre a equipe de saúde, como por exemplo, padronizando formas de prescrição e demais informações sobre medicamentos, para evitar erros de interpretação. No estudo de Paranaguá TTB, et al. (2014a), evidenciouse que as causas associadas à ocorrência de erros de medicação acentuam-se intervenção cirúrgica, período de internação hospitalar igual ou maior que quatro dias e a prescrição diária de três ou mais medicamentos.

Já os fatores que influenciam a omissão dos cuidados de enfermagem, de acordo com Valles JHH, et al. (2016), são atribuídos especialmente a fatores de recursos humanos, materiais e de comunicação, em que os recursos humanos é o fator mais relevante para a administração de enfermagem. Nesse sentido, a necessidade de se criar medidas de gestão que permitam consolidar os recursos humanos dentro das 
instituições de saúde, com a quantidade e competências especificas para dispensar uma atenção contínua, de acordo com as necessidades dos clientes, e evitar o cuidado omitido e o seu impacto nos resultados da atenção.

No que concerne a quedas em pacientes que ocorreram em 10,8\% dos casos notificados, segundo Sousa KAS (2014), são incidentes que continuam a acontecer com enorme frequência. Esta é uma realidade que deve ser considerada como um problema de gestão, e não do cliente, o qual precisa ser gerenciado.

O estudo identificou que o índice de quedas em hospitais de Belo Horizonte foi de 7,2 quedas por 1.000 pacientes hospitalizados. Assim, Pasa TS, et al. (2017), escrevem que utilizar ferramentas específicas na identificação de indivíduos com maior suscetibilidade de cair pode ser uma aliada na prevenção do incidente

De acordo Almeida RAR, et al. (2010), a prevenção de quedas deverá passar por uma rigorosa avaliação individual do risco, logo no momento da admissão do paciente na instituição. Frente a esse mesmo risco deverão ser empregadas guidelines que objetivem a sua minimização ou o controle dos fatores precipitantes. Enfim, terá que ser conduzida uma monitorização contínua ao longo da internação, notificando e identificando a ocorrência desse incidente, o aparecimento de novas condições de risco, de forma a adequar e/ou refazer as intervenções planeadas.

Em relação à falta de material médico hospitalar, foi observado em $8,4 \%$ dos eventos deste estudo. Resultados próximos ao de Paranaguá TTB, et al. (2014b), em que 13,8\% dos incidentes estavam relacionados à previsão e provisão de materiais, como a deficiência na reposição e manutenção tanto de materiais como de equipamentos.

Para assegurar atendimento de qualidade à população, é imprescindível melhor gerenciamento na aquisição de insumos, reduzir a falta de alguns e evitar o vencimento de outros. A necessidade de diminuir o desperdício de materiais de consumo médico-hospitalar (RAIMUNDO EA, et al., 2014).

Quanto ao setor de maior ocorrência de incidentes com as mães, destacam-se a clínica obstétrica e o préparto. Segundo Silva LA, et al. (2014), analisar os setores de maiores ocorrências de notificação de EA tornase importante por mostrar a gestão hospitalar, os principais setores que precisam de melhorias assistenciais, através de planos e estratégias de qualidade de trabalho.

Com relação aos recém-nascidos, boa parte dos incidentes ocorreram na UTIN. Segundo Novaretti MCZ, et al. (2014), alguns fatores são essenciais para colaborar a ocorrência de falhas nesses locais, como a complexidade dos casos, a necessidade de medidas de alto risco de forma rápida, a ausência de informações antecipadas sobre a saúde dos pacientes, realização de procedimentos diagnósticos e terapêuticos em frequência muito elevada, quando comparada a outros setores, e a utilização de um arsenal de medicamentos muito mais complexo em função da maior gravidade do quadro clínico inicial.

No que se refere às falhas que ocorreram com os $\mathrm{RN}$, além da identificação incorreta, foram encontrados outros incidentes mais frequentes, como flebite, omissão, LPP e extubação acidental. O que diverge do estudo de Lanzillotti LS, et al. (2015); e os 19 estudos incluídos na revisão sistemática conduzida pelos autores, evidenciaram que os incidentes com medicamentos como os mais comuns, sendo o grupo mais atingido, os mais vulneráveis: os RN pré-termo e de baixo peso. Corroborando com os resultados de Hoffmeister LV, et al. (2019), realizado na cidade de Porto Alegre e Morse ML, et al. (2011). A fase da assistência em que mais ocorreram incidentes e EA foi durante a internação $(75,5 \%)$. Esse resultado encontra-se em conformidade com estudos prévios, como nos achados Mendes W, et al. (2013) realizado em hospitais do Rio de Janeiro $(86,2 \%)$, Lorenzini E, et al. (2014) $(64,8 \%)$ no Sul do Brasil, Figueiredo ML, et al. (2018) (21\%) e Göttems LBD, et al. (2016) (11\%) no Distrito Federal.

Quanto o turno de maior acontecimento de EA, 62\% dos profissionais que notificaram ignoraram esta variável. No entanto, a literatura cientifica tem mostrado o turno em que mais aconteceram esses eventos, como no estudo de Lima CSP e Barbosa SFF (2015), no qual o turno de trabalho com maior número de casos notificados foi o noturno (39\%), resultado próximo ao de Figueiredo ML, et al. (2018) $(36,8 \%)$ e Silva LA, et al. $(2014)(40,2 \%)$. 
De acordo com Silva LA, et al. (2014), os profissionais de enfermagem que atuam em mais de um emprego por dia encontram-se em condições que podem facilitar para que os erros sejam acometidos, especialmente, no período da noite. Esses profissionais carregam posturas cansadas para o desenvolvimento do trabalho e terminam cometendo erros pela ausência de descanso e atenção.

A notificação nas fichas da instituição era de forma voluntária e anônima, uma vez que não é necessária autenticação para a equipe do hospital. Embora em $53 \%$ das notificações tenha sido ignorada a categoria profissional, a enfermagem foi a única categoria profissional que se identificou, pois $46,4 \%$ eram enfermeiros e apenas um $(0,6 \%)$ era técnico de enfermagem. A literatura científica tem reportado que os profissionais de enfermagem são responsáveis pela maioria das notificações. Como no estudo de Souza RF, et al. (2018), onde observaram que $78 \%$ das notificações foram realizadas por enfermeiros. Resultado semelhante ao de Ramos FO e Zuniga RAA (2018), em um estudo conduzido na Argentina, tendo $40.7 \%$ das notificações sido realizadas por essa categoria profissional e $52.7 \%$ em um hospital acadêmico terciário localizado no nordeste da Itália do estudo de Tricarico P, et al. (2017).

Já quanto à prevalência de incidentes notificados que ocorreram nos últimos dois anos, observou-se que o número de notificações aumentou com o decorrer dos anos, e houve um aumento de $36,2 \%$ de casos notificados no período de um ano. Corroborando com o estudo de Lorenzini E, et al. (2014), o crescimento do número total de casos notificados mostrou-se presente nas comparações no decorrer dos anos, havendo um crescimento médio entre 2008 a 2012 de 25,2\%. Figueiredo ML, et al. (2018) apresentou resultados semelhantes.

Entretanto, acredita-se que apesar da evolução temporal das notificações, há existência da subnotificação, pelo fato da notificação ocorrer de forma espontânea e voluntária. De acordo com Paranaguá TTB, et al. (2014b), os EA ainda são identificados rotineiramente no ambiente de trabalho. A pesquisa de Claro CM, et al. (2011), realizada no Estado de São Paulo em Campos do Jordão, evidenciou que a maior parte $(71,4 \%)$ dos profissionais percebem a existência de subnotificação de EA. Esses profissionais indicaram 115 motivos para esses comportamentos. Destacam-se a sobrecarga de trabalho $(25,2 \%)$, esquecimento $(22,6 \%)$, não valorização dos EA $(20,0 \%)$, sentimentos de medo $(15,7 \%)$, vergonha $(11,3 \%)$, entre vários outros.

A fim de superar as lacunas do conhecimento e expandir o compromisso de notificar e diminuir as falhas da prática profissional, e para superar as diferentes formas de comunicar ou omitir um incidente, é fundamental que a gestão hospitalar, juntamente com o apoio de seus profissionais, assuma condutas que fortaleçam essa cultura, e que possam ser executadas por meio de liderança comunicativa, sem punições e desenvolvimento pessoal. Estas são atribuições e competências desenvolvidas pela gestão para melhorar o relato (SIMAN AG, et al., 2017).

De acordo com Andrade LEL, et al. (2018), essas ações que visam melhoria da cultura de segurança em hospitais devem focar as dimensões "Frequência de EA notificados", "Conhecimento de segurança", "Feedback e comunicação sobre EA", "Resposta não punitiva para erros", "Dimensionamento de profissionais", "Trabalho em equipe entre unidades" e "Problemas em alterações de turno e mudanças entre unidades/serviços", visto que foram as áreas em que ocorreram os menores percentuais de respostas positivas analisadas pelo autor.

\section{CONCLUSÃO}

Os resultados reforçam a necessidade de estratégias, como de educação permanente da equipe de saúde, capacitação a partir de protocolos e guias de procedimentos para minimizar seus índices e estimular 0 monitoramento contínuo e preenchimento correto das fichas. A gestão hospitalar deve demonstrar apoio aos seus profissionais, desmistificando que esses incidentes não são um subproduto inevitável da assistência à saúde, mas passiveis de prevenção, através de uma cultura colaborativa, não punitiva, mas que colabora para a aprendizagem baseada nos próprios erros e valorização dos profissionais que as notificam. A atuação de enfermagem é fundamental para garantir a qualidade e segurança aos pacientes, que são vistas como prioridades da OMS. A enfermagem deve adotar medidas de prevenção e controle, através da identificação 
dos grupos de maior vulnerabilidade, uma vez que essa categoria profissional presta assistência direta e acompanha a clientela de perto, além do levantamento de um diagnóstico do ambiente de trabalho e as áreas de melhorias, atentando para os setores de maior ocorrência de incidentes notificados.

\section{REFERÊNCIAS}

1. ADEOYE IA, et al. Incidence, determinants and perinatal outcomes of near miss maternal morbidity in lle-Ife Nigeria: a prospective case control study. Bmc Pregnancy And Childbirth, 2013; 13(1): 1-10.

2. ADHIKARI NKJ. Patient safety without borders: measuring the global burden of adverse events. Bmj Quality \& Safety, 2013; 22(10): 798-801.

3. ALMEIDA RAR, et al. Quedas em doentes hospitalizados: contributos para uma prática baseada na prevenção. Revista de Enfermagem Referência, 2010; (2): 163-172.

4. ANACLETO TA, et al. Erros de medicação. Conselho Federal de Farmácia, 2010; 1-24.

5. ANDRADE LEL, et al. Cultura de segurança do paciente em três hospitais brasileiros com diferentes tipos de gestão. Ciência \& Saúde Coletiva, 2018; 23(1): 161-172.

6. BRASIL. Agência Nacional de Vigilância Sanitária. Gestão de Riscos e Investigação de Eventos Adversos Relacionados à Assistência à Saúde. Brasília: ANVISA, 2017.

7. CLARO CM et al. Eventos adversos em Unidade de Terapia Intensiva: percepção dos enfermeiros sobre a cultura não punitiva. Rev. esc. enferm. USP, 2011; 45(1): 167-172.

8. DAVID E, et al. Maternal near miss and maternal deaths in Mozambique: a cross-sectional, region-wide study of 635 consecutive cases assisted in health facilities of Maputo province. Bmc Pregnancy And Childbirth, 2014; 14(1): 1-8.

9. FASSINI P, HAHN GV. Riscos à segurança do paciente em unidade de internação Hospitalar: Concepções da equipe de enfermagem. Rev Enferm UFSM, 2012; 2(2): 290-299.

10. FIGUEIREDO ML, et al. Analysis of incidents notified in a general hospital. Revista Brasileira de Enfermagem, 2018; 71(1): 111-119.

11. GÖTTEMS LBD et al. A study of cases reported as incidents in a public hospital from 2011 to 2014. Revista da Escola de Enfermagem da Usp, 2016; 50(5): 861-867.

12. HOFFMEISTER LV, et al. Learning from mistakes: analyzing incidents in a neonatal care unit. Revista Latinoamericana de Enfermagem, 2019; 27:1-8.

13. LANZILLOTTI LS, et al. Adverse events and other incidents in neonatal intensive care units. Ciência \& Saúde Coletiva, 2015; 20(3):937-946.

14. LIMA CSP, BARBOSA SFF. Ocorrência de eventos adversos como indicadores de qualidade assistencial em unidade de terapia intensiva. Revista Enfermagem Uerj, 2015; 23(2): 222-228.

15. LORENZINI E, et al. Patient safety: analysis of the incidents notified in a hospital, in south of Brazil. Revista Gaúcha de Enfermagem, 2014; 35(2): 121-127.

16. MENDES W, et al. Características de eventos adversos evitáveis em hospitais do Rio de Janeiro. Revista da Associação Médica Brasileira, 2013; 59(5): 421-428.

17. MENDES W, et al. The assessment of adverse events in hospitals in Brazil. International Journal For Quality In Health Care, 2009; 21(4): 279-284.

18. MORSE ML, et al. Morbidade Materna Grave e Near Misses em Hospital de Referência Regional. Rev. bras. epidemiol., 2011; 14(2): 310-322.

19. MOURELLE JAF, et al. Reacciones adversas no descritas en embarazadas de Cuba. Rev Cubana Farm, 2015; 49(2): 291-303.

20. MUNHOZ OL. Profile of patients and incidents in a surgical clinic unit. J Nurs UFPE, 2018; 12(2): 416-23.

21. NOVARETTI MCZ, et al. Sobrecarga de trabalho da Enfermagem e incidentes e eventos adversos em pacientes internados em UTI. Revista Brasileira de Enfermagem, 2014; 67(5): 692-699.

22. OLIVEIRA TC. Eventos adversos e fatores associados em maternidades de ensino de Maceió, AL. Dissertação (Mestrado em enfermagem) - Universidade Federal de Alagoas, Maceió, 2018; 81 p.

23. PARANAGUÁ TTB, et al. Prevalence and factors associated with incidents related to medication in surgical patients. Revista da Escola de Enfermagem da Usp, 2014a; 48(1): 41-47.

24. PARANAGUÁ TTB et al. Incidentes: instrumento de gerenciamento da assistência para a segurança do paciente em pronto socorro. Revista eletrônica trimestral de enfermeira, 2014b; 13(34): 219-231.

25. PASA TS, et al. Risk assessment and incidence of falls in adult hospitalized patients. Revista Latino-americana de Enfermagem, 2017; 25: 1-8.

26. RAIMUNDO EA, et al. Logística de medicamentos e materiais em um hospital público do distrito federal. Rahis, 2015; 12(2): 61-69.

27. RAMOS FO, ZUÑIGA RAA. Underutilization of the reports of adverse events in na Argentine hospital. International Journal Of Risk \& Safety In Medicine, 2018; 29(3-4):159-162.

28. SILVA LA, et al. Notification of adverse events: characterization of events occurred in a hospital institution. J Nurs UFPE, 2014; 8(7): 3015-23.

29. SIMAN AG, et al. Ações de enfermagem para segurança do paciente em hospitais: revisão integrativa. Rev enferm UFPE, 2017; 11(2): 1016-1024. 
30. SOUSA KAS. Quedas de pacientes adultos em um Hospital Público de Ensino, MG. Dissertação (Mestrado em enfermagem) - Escola de Enfermagem da Universidade Federal de Minas Gerais, Belo Horizonte, $2014 ; 106$ p.

31. SOUSA P, MENDES W. Segurança do paciente: conhecendo os riscos nas organizações de saúde. Rio de Janeiro: Fiocruz, 2014; 452p.

32. SOUZA FT, et al. Percepção da enfermagem sobre os fatores de risco que envolvem a segurança do paciente pediátrico. Revista de Enfermagem da UFSM, 2014; 4(1): 152-162.

33. SOUZA RF, et al. Eventos adversos na unidade de terapia intensiva. Rev enferm UFPE, 2018; 12(1): $19-27$.

34. TASE TH. Evaluation of the risk of misidentification of women in a public maternity hospital. Revista Brasileira de Enfermagem, 2018; 71(1): 120-125.

35. TRICARICO P, et al. Professional attitudes toward incident reporting: can we measure and compare improvements in patient safety culture?. International Journal For Quality In Health Care, 2017; 29(2): 243-249.

36. VALLES JHH, et al. Nursing care missed in patients at risk of or having pressure ulcers. Revista Latino-americana de Enfermagem, 2016; 24: 1-8.

37. WORLD HEALTH ORGANIZATION. World Alliance for Patient Safety, Taxonomy: The Conceptual Framework for the International Classification for Patient Safety: final technical report. Genebra: WHO, 2009. 